\title{
The transcription factor E2F2 regulates tumor development
}

\author{
Qianfan Liu ${ }^{1}$, Zou Xiang ${ }^{2}$, Haitao Zhu $^{1 *}$ \\ ${ }^{\prime}$ Department of Hepatobiliary Surgery, Affiliated Hospital of Guizhou Medical University, Guiyang, Guizhou 550004, China; \\ ${ }^{2}$ Department of Health Technology and Informatics, Faculty of Health and Social Sciences, The Hong Kong Polytechnic University, \\ Hung Hom, Kowloon, Hong Kong, China.
}

\begin{abstract}
Cell cycle-related E2F transcription factor $2(\mathrm{E} 2 \mathrm{~F} 2)$ is a member of the E2F transcription factor family. E2F2 is implicated in tumorigenesis as it functions as a nuclear transcription factor responsible for regulating cell cycle progression to maintain genomic integrity. In recent decades, substantial experimental evidence has associated the function of E2F2 with tumor promotion. However, this transcription factor has also been revealed to be capable of exerting tumor suppressive activities in certain tumor models. In this review, we discuss recent developments in research that focus on E2F2 with respect to tumorigenesis. We will particularly address the dynamic regulatory roles of microRNA (miRNA) on the expression of E2F2 as various miRNA species have been confirmed to have complementary binding sites in the 3' untranslated region of E2F2.
\end{abstract}

Keywords: E2F2, tumor, miRNA, transcription factor

\section{OVERVIEW OF E2F2}

In 1986, Kovesdi and co-workers discovered a group of transcription factors which interact with the adenovirus E2 promoter, an ElA inducible promoter from the nuclear extracts of adenovirus-infected cells ${ }^{[1]}$. The first family member, named E2Fl, the gene product of which demonstrates the properties of E2F, was reported by Helin et al. in $1992^{[2]}$. Next, another novel protein in this family, E2F2 was cloned by Ivey-Hoyle et al. in 1993 by screening a HeLa cDNA library with a DNA probe derived from the DNA-binding domain of $\mathrm{E} 2 \mathrm{Fl} 1^{[3]}$. E2F2 and E2F1 exhibit $46 \%$ amino acid identity ${ }^{[4]}$. E2F2, which contains 10 exons coding for 438 amino acids, maps to 1 p36.12 on the short arm of human chromosome 1. Since then 6 additional E2F family members with a high degree of homology have been discovered, and up to date altogether 8 homologs, namely, E2F1 to E2F8, have been described ${ }^{[5]}$. They share a number of evolutionarily conserved domains, including a highly conserved DNA-binding domain, a dimerization domain which determines their interaction with differentiation-regulated transcription factor proteins (DP), and an acidic amino acid-enriched transactivation domain within which a tumor suppressor protein association domain is embedded.

$\mathrm{E} 2 \mathrm{~F} 2$ is an important member of the E2F family of transcription factors that are capable of exerting regulatory roles in dynamic cellular processes including differentiation, development, proliferation and apoptosis ${ }^{[6]}$. E2F2 functions as a nuclear transcription factor responsible for regulating mitosis to maintain genomic integrity. Activation of E2F2 promotes the Gl-to S-phase transition in cell cycling, indicating that this molecule is one of the key regulators in cell cycle progression. Recently, substantial experimental evidence has associated the function of E2F2 with tumor

*Correspondence to: Haitao Zhu, Department of Hepatobiliary Surgery, Affiliated Hospital of Guizhou Medical University, Guiyang, Guizhou 550004, China. Tel: +86-13885159100. E-mail: 754667317@qq.com.

The authors declared no conflict of interests. 
promotion in addition to many physiological processes regulated by this transcription factor.

Studies resolving the interactions between E2F transcription factors and retinoblastoma protein $(\mathrm{pRb})$, a tumor suppressor protein, have contributed to the elucidation of the common function of E2F proteins. $\mathrm{pRb}$ is the founding member of a protein family of tumor suppressors that demonstrates a crucial role in the regulation of G1-to S-phase transition. Loss of its control over cell cycle progression is one of the mechanisms that account for tumorigenesis ${ }^{[7]}$. These proteins fold into a binding pocket-like structure and are thus collectively addressed as the pocket protein family ${ }^{[8]}$. $p R b$ is capable of regulating entry into $\mathrm{S}-$ phase through a direct interaction between its large pocket domain and the transactivation domain of E2F members (e.g., E2F1, E2F2, and E2F3) resulting in the repression of E2F-dependent transcription of Sphase-promoting genes including DNA polymerases. In G0 and early Gl, pRb physically interacts with E2F factors, thereby blocking the transactivation domain of E2F. At the onset of S-phase, E2F is released from the $\mathrm{pRb}-\mathrm{E} 2 \mathrm{~F}$ complex as a result of phosphorylation of $\mathrm{pRb}$ which is driven by cyclin $\mathrm{D}$ and its associated cyclin-dependent kinases (CDK). Dissociation of E2F from $\mathrm{pRb}$ exposes E2F-binding sites for the promoters of cell-cycle proteins, resulting in cell cycle progression $^{[7,9]}$. Therefore, E2F, through collaboration with $\mathrm{pRb}$, is required for the full execution of cell cycle progression and this family of transcription factors is involved in the regulation of tumor development.

Loss of E2F2 is generally correlated with decreased proliferation of tumor cells, which is consistent with its role in promoting the transcription of quite a number of cell cycle-promoting genes. Paradoxically, depending on the context, mice with mutations in E2F2 can exhibit increased cancer incidence ${ }^{[10,11]}$. This paradox is also reflected in certain human cancers, such as in human glioma ${ }^{[12]}$, breast cancer ${ }^{[13]}$, colon cancer ${ }^{[14]}$, and gastric cancer ${ }^{[15]}$, whereby it is revealed that E2F2 exerts tumor suppressive or prognostic activities. We will discuss these models in further detail below.

Interplay between microRNA (miRNA) and E2F members including E2F2 has been an attractive area of research in tumor biology. miRNAs are a family of 21-to 25-nucleotide, noncoding small RNAs that primarily function as gene regulators. Elucidation of the roles of miRNAs in tumorigenesis has advanced our understanding in the complex mechanisms of cancer development in recent years ${ }^{[16,17]}$. It is widely accepted that altered miRNA expression impacts tumour growth, progression and metastasis. In this review, we will also discuss the interaction between miRNA and
E2F2 as well as the functional consequence of this interaction in a number of tumor models. In addition to regulating cell cycle progression, $\mathrm{pRb}$ has been implicated in various cellular events, including differentiation, apoptosis, motility, cartilage development, and vascularization ${ }^{[18]}$. Likewise, E2F proteins, including E2F2, also exert dynamic regulatory roles in other pathophysiologic settings in addition to cancer, which is not the focus of this review.

\section{THE REGULATORY EFFECTS OF E2F2 ON TUMOR DEVELOPMENT}

\section{Leukemia}

Leukemia collectively refers to hematopoietic stem cell-initiated heterogeneous blood cancers. Leukemia usually originates in the bone marrow and results in abnormal blood cell proliferation in both the bone marrow and peripheral blood. The uncontrolled growth of blood cancer cells outgrow normal blood cells causing symptoms that include easy bleeding or bruising, persistent fatigue, frequent fevers, and an increased risk of infections. Leukemia is grouped by how rapidly the disease develops, i.e., acute or chronic, as well as by the major type of blood cells involved, i.e., lymphocytes or myelocytes. Thus, leukemia is mainly divided into four main types: acute lymphoblastic leukemia (ALL), acute myeloid leukemia (AML), chronic lymphocytic leukemia (CLL), and chronic myeloid leukemia (CML) ${ }^{[19]}$. The most common types of leukemia in adults are AML and CLL, followed by CML and ALL. Despite the fact that approximately 10 times more adults than children develop leukemia, it is the most prevalent cancer among children, with ALL accounting for approximately 75 percent of all childhood leukemia cases. The overall yearly incidence for leukemia has increased modestly in recent decades, while overall mortality rates have fallen since the $1990 \mathrm{~s}{ }^{[20]}$.

Increase in steady-state E2F2 protein is correlated with the level of Tax protein of human T-cell leukemia virus type I, a transcriptional activator implicated in cellular transformation and leukaemia development ${ }^{[21,22]}$. The requirement of myeloid translocation gene 16 (MTG16) for maintenance of haematopoietic stem cell quiescence is probably associated with its capacity to suppress the expression of a number of key cell cycle regulators including E2F2 ${ }^{[23]}$. Although enhanced leukemogenesis is observed in E2F1/E2F2 double knockout mice, this is found to be primarily due to the weakened capacity of double knockout progenitor cells to compete with Bcr-Abl-expressing double knockout progenitors rather than cell autonomous ef- 
fects of E2F2 loss ${ }^{[24]}$. In conclusion, experimental evidence supports the cancer promoting effect of $\mathrm{E} 2 \mathrm{~F} 2$ in leukemia. However, the regulatory role of E2F2 in leukemia has not been thoroughly investigated. Therefore, this is an area that awaits further experimental exploitation and input.

\section{Glioma}

Glioma refers to a type of tumor that starts in the glial cells of the brain or spinal cord. Glioblastoma, also known as glioblastoma multiforme (GBM), is the most frequent, aggressive and deadly primary malignant tumor of the central nervous system, accounting for $60 \%$ 70\% of all gliomas and $15 \%$ of all intracranial tumors. No satisfactory treatment for this ferocious type of malignancy is available, and the median survival time is less than 16 months following initial diagnosis ${ }^{[25]}$. Management of glioblastoma remains palliative as optimal treatment including surgery and chemotherapy only results in a median survival of less than one year. Only about $2 \%$ of patients survive three years.

Network-based survival analysis reveals that high expression of E2F2 is correlated with poor prognosis. By exploiting data in the Chinese Glioma Genome Atlas (CGGA) database, Zhang et al. found that expression levels of E2F2 in glioma are higher than normal brain tissues, and miRNAs play crucial roles in regulating the tumorigenesis of glioma. Furthermore, they revealed that miR-218 inhibits the growth and metabolism of gliomas by directly targeting E2F2. Reduced expression levels of miR-218 are observed in human glioblastoma tissues. In contrast, enforced expression of miR-218 in human glioblastoma cell lines arrests cell cycle progression in the Gl-phase, thus profoundly suppressing cell proliferation. Both glucose consumption and lactate production by glioblastoma cells are also inhibited with enhanced expression of miR-218. It is thus proposed that miR-218, a repressor of E2F2 in glioblastoma, may be exploited as a therapeutic target ${ }^{[26]}$. Two binding sites for miR$125 \mathrm{~b}$, which inhibits the proliferation of glioblastoma stem cells, are identified in E2F2, suggesting the involvement of E2F2 in miR-125b-mediated regulation of glioblastoma development ${ }^{[27]}$. Low expression of PPAR $\alpha$ is correlated with poor prognosis of glioma and GBM patients, and the mechanism may be associated with PPAR $\alpha$-induced expression of miR214 which suppresses its target E2F2 ${ }^{[28]}$. One more piece of evidence showing the regulation of E2F2 by miRNA in glioma cells comes from the study demonstrating that the Let- 7 miRNA precursor inhibits the malignant development of glioma cells via down-reg- ulation of $\mathrm{E} 2 \mathrm{~F} 2{ }^{[29]}$. In contrast to the tumor promoting activity of E2F2, it is also observed that enforced expression of E2F2 enhances p53-mediated anti-cancer effect in human glioma cells ${ }^{[12]}$.

\section{Lung cancer}

Being the most common cause of cancer deaths worldwide, lung cancer is one of the most aggressive tumors, with very low life expectancy. Lung cancer includes two broad histological subtypes: smallcell lung cancer (SCLC), which is the cause of $15 \%$ of cases, and non-small-cell lung cancer (NSCLC), which accounts for $85 \%$ of cases. NSCLC is further divided into adenocarcinoma, squamous-cell carcinoma, and large-cell carcinoma ${ }^{[30]}$. Surgery remains the standard of treatment for early-stage NSCLC. However, not all patients benefit from tumor resection by surgery as some patients develop recurrence after surgery. Biomarkers that help determine clinical staging and predict treatment outcome are therefore warranted.

As mentioned above, E2F2 promotes lung metastasis in breast cancer ${ }^{[13,31]}$. Chen et al. analyzed lung samples from 86 NSCLC patients by immunohistochemistry as well as lung tissues from 8 NSCLC patients by Western blotting for E2F2 expression ${ }^{[32]}$. Both approaches consistently confirmed that E2F2 expression is markedly increased in NSCLC tissues compared with adjacent normal tissue controls. Furthermore, E2F2 expression is correlated with tumor cell proliferation and poor patient prognosis. Of note, E2F2 expression is closely correlated with clinical stage and tumor size. Knockdown of E2F2 expression reduces the viability and colony formation capacity of NSCLC cells ${ }^{[32]}$. Based on exploitation of the Oncomine, GEPIA, Kaplan-Meier(KM) Plotter, and cBioPortal databases, Sun et al. made interesting observations regarding E2F family proteins ${ }^{[33]}$. Expression levels of $\mathrm{E} 2 \mathrm{~F} 2$, among a group of other $\mathrm{E} 2 \mathrm{~F}$ factors, are higher in lung adenocarcinoma and squamous cell lung carcinoma tissues than in normal lung tissues. The expression levels of E2F2, among certain other E2F family members, are positively correlated with advanced tumor staging. High transcription levels of a panel of E2F members including E2F2 are correlated with low relapse-free survival in all lung cancer patients. Taken together, these findings support $\mathrm{E} 2 \mathrm{~F} 2$, together with E2F1, E2F4, E2F5 and E2F8, as new biomarkers for the prognosis of lung cancer ${ }^{[33]}$. Based on bioinformatics analysis, E2F2 expression is found to be significantly correlated with NSCLC patient gender and tumor size, with higher expression levels in males over females and higher expression in 
later stages. Furthermore, overexpression of E2F2 is correlated with poor prognosis ${ }^{[34]}$. Through analyzing miRNA expression in 47 lung cancer biopsies, Feliciano et al. reported that expression of miR-99a, which is capable of downregulating E2F2 by direct binding to the 3' untranslated region ( 3 '-UTR) of E2F2, is correlated to decreased proliferation, cell cycle arrest and apoptosis of a panel of lung cancer cell lines ${ }^{[35]}$. It is recently revealed that circular RNA circPVTl promotes tumor proliferation and invasion through sponging miR-125b and activating E2F2 signaling in NSCLC ${ }^{[36]}$, as E2F2 is a target of miR-125b which has a protective role in some cancers, e.g., glioblastoma as mentioned above $^{[27]}$.

\section{Breast cancer}

Breast cancer is the most common cancer in women worldwide and the second most common cancer overall after the lung cancer. Although steady increases in the incidence of breast cancer in western countries were recorded toward the end of last century, which is believed to be primarily due to increased screening, reproductive pattern changes, and wide use of menopausal hormonal therapy, breast cancer incidence has declined since the early $2000 \mathrm{~s}{ }^{[37]}$. However, breast cancer still accounts for more than 268,000 new cases and 40,000 fatalities in the US alone in $2019^{[38]}$. Breast cancer incidence rates in less developed areas of the world continue to increase, probably reflecting changing reproductive patterns as well as increased screening ${ }^{[37]}$.

Based on the datasets in Oncomine and the Cancer Genome Atlas, E2F2, together with a group of other $\mathrm{E} 2 \mathrm{~F}$ factors including E2F1, E2F3, E2F5, E2F7 and $\mathrm{E} 2 \mathrm{~F} 8$, is overexpressed at the mRNA level in patients with breast cancer ${ }^{[39]}$. Rennhack et al. found that loss of E2F1 or E2F2 leads to reduced metastasis in mouse mammary tumor virus (MMTV)-Neu mice which are prone to mammary tumorigenesis. They observed that $73 \%$ of the mice with mammary tumor induction developed metastasis to the lungs, which stands in clear contrast to the reduced percentage of $35 \%$ when $\mathrm{E} 2 \mathrm{~F} 2$ is deleted in similar mice. Importantly, the model is supported by analyzing human HER2-positive cancer patients ${ }^{[00]}$. In the mouse MMTV-polyomavirus middle T oncoprotein (PyMT) model of metastatic breast cancer, it is noted that deficiency in E2F2 strikingly reduces tumor metastasis ${ }^{[31]}$. It is reported that E2F2, with three binding sites, is a direct target of miR-4319 which is capable of suppressing breast tumor initiation and metastasis ${ }^{[41]}$. Breast cancer cell-matrix adhesion and outgrowth are profoundly inhibited when E2F2 levels are lowered ${ }^{[42]}$. Knockdown of E2F2 expression results in disruption of estrogen receptor-positive breast cancer cell proliferation ${ }^{[43]}$. Activating protein-1 (AP-1), a transcription factor that regulates gene expression in response to a variety of stimuli, is involved in promoting breast cancer cell growth, and suppression of AP-1 expression is correlated with reduced expression of E2F1 and E2F2 ${ }^{[44]}$. E2F2 deficiency suppresses breast cancer metastasis and hence results in reduced circulating tumor cells ${ }^{[45]}$. All these cases are consistent with the major role of E2F2 as a tumor promoter. However, studies pointing to the contrary have also been reported. Loss of E2F2 promotes lung metastasis in breast cancer, whereas downregulating the expression of the tumor suppressor gene PTPRD suppresses lung metastasis, suggesting antagonism between E2F2 and PTPRD ${ }^{[13]}$. It is also been revealed that genetic deletion of E2F2 accelerates mammary tumorigenesis driven by Myc-overexpression ${ }^{[46]}$.

\section{Gastric cancer}

Gastric cancer develops from the lining of the stomach and remains one of the most common and deadly human neoplasms, with an estimated annual global death toll of up to $800,000^{[47]}$. More than $70 \%$ of these cases occur in developing countries, and China is one of the countries with the highest incidences of gastric cancer, accounting for over $40 \%$ of all new gastric cancer cases in the world ${ }^{[48,49]}$. Most cases of stomach cancer are gastric carcinomas, which can be further divided into a number of subtypes including gastric adenocarcinomas ${ }^{[50]}$. Surgery remains the major choice of treatment for stomach cancer, supplemented with chemotherapy and radiation therapy ${ }^{[51]}$. Most gastric cancer patients are asymptomatic in the early stages which makes timely diagnosis difficult. Therefore, diagnostic markers which help to reveal early-stage asymptomatic cases are desperately needed.

miR-31 has been found reduced in gastric cancer and the expression of miR-31 is negatively correlated with poor tumor differentiation, lymph node metastasis, advanced tumor staging and worse overall survival of gastric cancer patients. Interestingly, E2F2 is the direct target of miR-31. Consistently, E2F2 expression, which is inversely correlated with miR31 expression, is upregulated in gastric cancer tissues. Furthermore, knockdown of E2F2 expression mimics the antitumor activity of miR-31 in gastric cancer cells, while the ectopic expression of E2F2 can rescue miR-31-mediated inhibition in the growth of gastric cell lines. Taken together, these results demonstrate that miR-31 acts as a crucial tumor suppressor by inhibiting E2F2 expression ${ }^{[52]}$. miR-26a, which has a target site in the 3'-UTR of E2F2, is downregulated in 
chemotherapy-resistant gastric cancer cells. Furthermore, knockdown of E2F2 sensitizes gastric cancer cells to chemotherapy ${ }^{[53]}$. These results suggest that miR-26a might improve the sensitivity of gastric cancer cells to chemotherapy through targeting E2F2.

Despite these models demonstrating the gastric cancer promoting activity of E2F2, the antitumor activity of E2F2 has also been observed. By using the KM Plotter database, which has been established using gene expression data and survival information from the Gene Expression Omnibus (GEO), Manicum and co-workers ${ }^{[15]}$ reported that increased expression of E2F2, together with E2F5, E2F6 and E2F7, is correlated with favorable overall survival, especially for late-stage gastric cancer patients. These results provide insight into the controversial prognostic function of E2F in gastric cancer. Although the results should be further verified in clinical trials, these findings suggest E2F2 may be a favorable prognostic predictor for evaluating the efficacy of therapeutic drugs in the treatment of gastric cancer.

\section{Other malignancies}

Evidence for the involvement of E2F2 in the regulation of other types of cancers has also been steadily accumulated. Chen et al. reported that E2F2, a target of miR-365, is highly expressed in cholangiocarcinoma tissues and cell lines, whereas miR-365 is downregulated in cholangiocarcinoma tissues compared with adjacent normal tissues. In an experimental model, overexpression of miR-365, which suppresses the expression of E2F2, is capable of inhibiting cholangiocarcinoma cell proliferation and drives apoptosis of these cancer cells. This finding is consistent with the clinically observed inverse correlation between the expression levels of E2F2 and miR-365 in cholangiocarcinoma tissues. These data suggest that the inhibition of E2F2 activity may benefit the treatment of cholangiocarcinoma ${ }^{[54]}$. Similar to the protective role of miR-218 revealed in glioma ${ }^{[26]}$, it is also reported that overexpression of miR-218 and miR-520a can inhibit proliferation of hepatocellular carcinoma cells. miR-218 and miR-520a are observed to negatively regulate $\mathrm{E} 2 \mathrm{~F} 2$ expression despite the fact that $\mathrm{E} 2 \mathrm{~F} 2$ is a direct target of miR-218, but not miR-520a. These results indicate that $\mathrm{E} 2 \mathrm{~F} 2$ is a critical tumor promoting molecule via which miR-218 and miR-520a regulate the development of hepatocellular carcinoma directly or indirectly, respectively ${ }^{[5]}$. Another miRNA, miR490-5p, has also been recently implicated in the suppression of hepatocellular carcinoma through downregulating E2F2 expression ${ }^{[56]}$.

Differentiation antagonizing nonprotein coding
RNA (DANCR), a type of long non-coding RNA (ncRNA), is profoundly upregulated in pancreatic cancer tissues as DANCR functions as a molecular sponge for miR-214-5p that inhibits E2F2 expression ${ }^{[57]}$. It is reported that human papillomavirus (HPV)16 E6 oncoprotein is capable of downregulating miR-496, which targets the 3' UTR of E2F2, providing a mechanism which underlies the role of HPV16 infection in oropharyngeal cancer ${ }^{[58]}$. Lower expression levels of miR-125a are observed in osteosarcoma tissues and miRNA-125a is found to be able to regulate tumor cell proliferation via targeting $\mathrm{E} 2 \mathrm{~F} 2{ }^{[59]}$.

Two more examples supporting E2F2 as a tumor suppressor come from studies addressing colon cancer and clear cell renal cell carcinoma. E2F2 is shown to inhibit the growth and proliferation of colon cancer cell lines ${ }^{[14]}$. In this study, it is worth pointing out that miR-31, which can directly target E2F2, promotes the proliferation of colon cancer cells. This stands in clear contrast to the above mentioned miR-31-mediated tumor suppression in gastric cancer ${ }^{[52]}$. Gao et al. reported that miR-155 functions as a tumor-enhancer by suppressing E2F2 in clear cell renal cell carcinoma, suggesting the tumor suppressive effect of E2F2 in this renal malignancy ${ }^{[60]}$.

\section{SUMMARY AND PERSPECTIVES}

As a member of the E2F family of transcription factors, E2F2 plays a critical role in the regulation of cell cycling, which has a profound impact on tumor initiation and growth. The potential of E2F2 in regulating tumor development is dynamic and contextdependent. In different cancers or during different tumor developmental stages of the same cancer, this transcription factor may exert distinct functions. Of note, E2F2 not only has many downstream targets for initiating gene expression, thereby modulating tumor development, but also is subject to the regulation of various upstream signals such as $\mathrm{pRb}$ and miRNA (Fig.1). The enigmatic roles of E2F2 on the promotion and suppression of different tumors await further clarification. It is possible that certain non-canonical roles of E2F2, i.e., activities other than cell cycle regulation, have not been revealed. Future research is warranted to fill the gaps in our understanding of the precise regulatory mechanisms of E2F2 as well as to elucidate the complex relationship between E2F2 and other E2F family members and tumor regulatory molecules. Targeting E2F2 may facilitate the rational design of cutting-edge diagnostic tools with better clinical relevance and novel anticancer therapies with higher efficacy. 


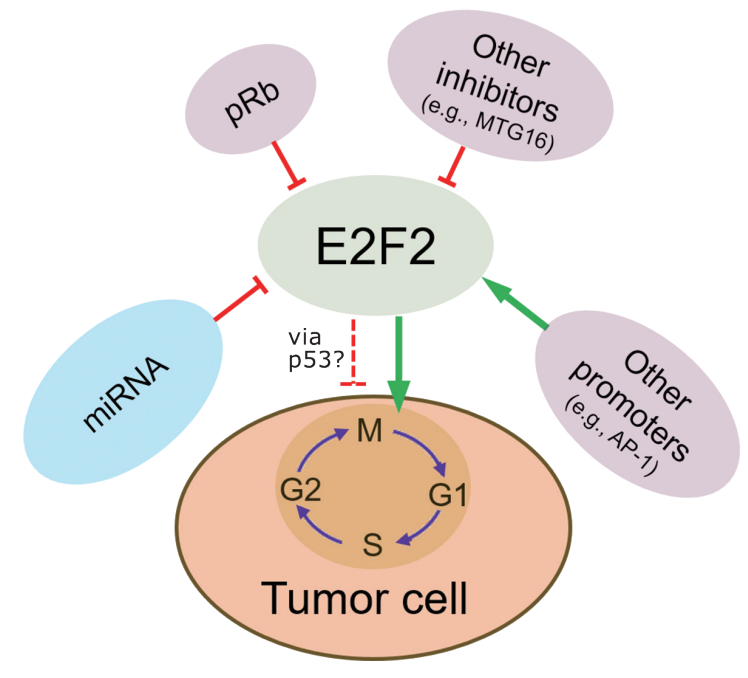

Fig. 1 Regulation of tumor development by E2F2 and associated partners. This schematic diagram illustrates the possible interactions between the transcription factor E2F2 and its regulatory partners for impacting tumor development. Overwhelming experimental evidence suggests that E2F2 promotes tumor development by stimulating cell cycle progression (green pointed arrow). However, some studies suggest an opposite, suppressive effect of E2F2 on tumor cells (red dashed blunt arrow), possibly via enhancing p53-mediated anti-cancer effect (ref 12). Various microRNA (miRNA) species, which have been confirmed to have complementary binding sites in the 3' untranslated region of E2F2, are capable of exerting inhibitory effects on E2F2(red blunt solid arrow). E2F2 is also subject to an inhibitory regulation by other molecules such as retinoblastoma protein (pRb) and MTGl6 (red blunt solid arrows). Factors (e.g., AP-1) that may upregulate E2F2 have also been reported (green pointed arrow). G1-S-G2-M, respective cell cycle phases; MTG16, myeloid translocation gene 16; AP1 , activating protein-1.

\section{Acknowledgments and funding}

This work is supported by National Natural Science Foundation of China (81960429) and Guizhou Province Science and Technology Project ([2018]5779-31).

\section{References}

[1] Kovesdi I, Reichel R, Nevins JR. Identification of a cellular transcription factor involved in E1A transactivation[J]. Cell, 1986, 45(2): 219-28.

[2] Helin K, Lees JA, Vidal M, et al. A cDNA encoding a pRB-binding protein with properties of the transcription factor E2F[J]. Cell, 1992, 70(2): 337-50.

[3] Ivey-Hoyle M, Conroy R, Huber HE, et al. Cloning and characterization of $\mathrm{E} 2 \mathrm{~F}-2$, a novel protein with the biochemical properties of transcription factor E2F[J]. Mol Cell Biol, 1993, 13(12): 7802-12.

[4] Joyce NC, Harris DL, Mc Alister JC, et al. Effect of overexpressing the transcription factor E2F2 on cell cycle progression in rabbit corneal endothelial cells[J]. Invest Ophthalmol Vis Sci, 2004, 45(5): 1340-8.
[5] Tsantoulis PK, Gorgoulis VG. Involvement of E2F transcription factor family in cancer[J]. Eur J Cancer, 2005, 41(16): 2403-14.

[6] Muller H, Bracken AP, Vernell R, et al. E2Fs regulate the expression of genes involved in differentiation, development, proliferation, and apoptosis[J]. Genes Dev, 2001, 15(3): 267-85.

[7] Dick FA, Goodrich DW, Sage J, et al. Non-canonical functions of the RB protein in cancer[J]. Nat Rev Cancer, 2018, 18(7): 442-51.

[8] Balciunaite E, Spektor A, Lents NH, et al. Pocket protein complexes are recruited to distinct targets in quiescent and proliferating cells[J]. Mol Cell Biol, 2005, 25(18): 8166-78.

[9] Uchida C. Roles of $\mathrm{pRB}$ in the regulation of nucleosome and chromatin structures[J]. Biomed Res Int, 2016, 2016: 5959721.

[10] Yamasaki L, Jacks T, Bronson R, et al. Tumor induction and tissue atrophy in mice lacking E2F-1[J]. Cell, 1996, 85(4): 537-48.

[11] Zhu JW, Field SJ, Gore L, et al. E2F1 and E2F2 determine thresholds for antigen-induced $\mathrm{T}$-cell proliferation and suppress tumorigenesis[J]. Mol Cell Biol, 2001, 21(24): 8547-64.

[12] Mitlianga PG, Kyritsis AP, Gomez-Manzano C, et al. Co-expression of E2F-2 enhances the p53 anti-cancer effect in human glioma cells[J]. Int J Oncol, 2001, 18(2): $343-7$.

[13] Yuwanita I, Barnes D, Monterey MD, et al. Increased metastasis with loss of E2F2 in Myc-driven tumors[J]. Oncotarget, 2015, 6(35): 38210-24.

[14] Li T, Luo W, Liu K, et al. miR-31 promotes proliferation of colon cancer cells by targeting E2F2[J]. Biotechnol Lett, 2015, 37(3): 523-32.

[15] Manicum T, Ni F, Ye Y, et al. Prognostic values of E2F mRNA expression in human gastric cancer[J]. Biosci Rep, 2018, 38(6): BSR20181264.

[16] Pedroza-Torres A, Romero-Cordoba SL, Justo-Garrido $\mathrm{M}$, et al. MicroRNAs in tumor cell metabolism: Roles and therapeutic opportunities[J]. Front Oncol, 2019, 9: 1404.

[17] Conti I, Varano G, Simioni C, et al. miRNAs as influencers of cell-cell communication in tumor microenvironment[J]. Cells, 2020, 9(1): 220.

[18] Bursch W, Karwan A, Mayer M, et al. Cell death and autophagy: cytokines, drugs, and nutritional factors[J]. Toxicology, 2008, 254(3): 147-57.

[19] Schnatter AR, Rosamilia K, Wojcik NC. Review of the literature on benzene exposure and leukemia subtypes[J]. Chem Biol Interact, 2005 ,153-154: 9-21.

[20] Hermine O, Ramos JC, Tobinai K. A review of new findings in adult T-cell leukemia-lymphoma: A focus on current and emerging treatment strategies[J]. Adv Ther, 2018, 35(2): 135-52.

[21] Neuveut C, Low KG, Maldarelli F, et al. Human T-cell leukemia virus type 1 Tax and cell cycle progression: role of cyclin D-cdk and p110Rb[J]. Mol Cell Biol, 1998, 18(6): 3620-32. 
[22] Azran I, Schavinsky-Khrapunsky Y, Aboud M. Role of Tax protein in human T-cell leukemia virus type-I leukemogenicity[J]. Retrovirology, 2004, 1: 20.

[23] Fischer MA, Moreno-Miralles I, Hunt A, et al. Myeloid translocation gene 16 is required for maintenance of haematopoietic stem cell quiescence[J]. EMBO J, 2012, 31(6): 1494-505.

[24] Bilousova G, Marusyk A, Porter CC, et al. Impaired DNA replication within progenitor cell pools promotes leukemogenesis[J]. PLoS Biol, 2005, 3(12): e401.

[25] Wick W, Platten M. Understanding and treating glioblastoma[J]. Neurol Clin, 2018, 36(3): 485-99.

[26] Zhang Y, Han D, Wei W, et al. MiR-218 inhibited growth and metabolism of human glioblastoma cells by directly targeting E2F2[J]. Cell Mol Neurobiol, 2015, 35(8): 1165-73.

[27] Wu N, Xiao L, Zhao X, et al. miR-125b regulates the proliferation of glioblastoma stem cells by targeting E2F2[J]. FEBS Lett, 2012, 586(21): 3831-9.

[28] Gao Y, Han D, Sun L, et al. PPARalpha regulates the proliferation of human glioma cells through miR-214 and E2F2[J]. Biomed Res Int, 2018, 2018: 3842753.

[29] Song H, Zhang Y, Liu N, et al. Let-7b inhibits the malignant behavior of glioma cells and glioma stem-like cells via downregulation of E2F2[J]. J Physiol Biochem, 2016, 72(4): 733-44.

[30] Oser MG, Niederst MJ, Sequist LV, et al. Transformation from non-small-cell lung cancer to small-cell lung cancer: molecular drivers and cells of origin[J]. Lancet Oncol, 2015, 16(4): el65-72.

[31] Hollern DP, Honeysett J, Cardiff RD, et al. The E2F transcription factors regulate tumor development and metastasis in a mouse model of metastatic breast cancer[J]. Mol Cell Biol, 2014, 34(17): 3229-43.

[32] Chen L, Yu JH, Lu ZH, et al. E2F2 induction in related to cell proliferation and poor prognosis in non-small cell lung carcinoma[J]. Int J Clin Exp Pathol, 2015, 8(9): 10545-54

[33] Sun CC, Zhou Q, Hu W, et al. Transcriptional $\mathrm{E} 2 \mathrm{~F} 1 / 2 / 5 / 8$ as potential targets and transcriptional E2F3/6/7 as new biomarkers for the prognosis of human lung carcinoma[J]. Aging (Albany NY), 2018, 10(5): 973-87.

[34] Gao Z, Shi R, Yuan K, et al. Expression and prognostic value of E2F activators in NSCLC and subtypes: a research based on bioinformatics analysis[J]. Tumour Biol, 2016, 37(11): 14979-87.

[35] Feliciano A, Garcia-Mayea Y, Jubierre L, et al. miR-99a reveals two novel oncogenic proteins E2F2 and EMR2 and represses stemness in lung cancer[J]. Cell Death Dis, 2017, 8(10): e3141.

[36] Li X, Zhang Z, Jiang H, et al. Circular RNA circPVT1 promotes proliferation and invasion through sponging miR-125b and activating E2F2 signaling in non-small cell lung cancer[J]. Cell Physiol Biochem, 2018, 51(5): 2324-40.

[37] Fahad Ullah M. Breast cancer: Current perspectives on the disease status[J]. Adv Exp Med Biol, 2019, 1152: 5164.

[38] Siegel RL, Miller KD, Jemal A. Cancer statistics, 2019[J]. CA Cancer J Clin, 2019, 69(1): 7-34.

[39] Li Y, Huang J, Yang D, et al. Expression patterns of E2F transcription factors and their potential prognostic roles in breast cancer[J]. Oncol Lett, 2018, 15(6): 9216-30.

[40] Rennhack J, Andrechek E. Conserved E2F mediated metastasis in mouse models of breast cancer and HER2 positive patients[J]. Oncoscience, 2015, 2(10): 867-71.

[41] Chu J, Li Y, Fan X, et al. MiR-4319 suppress the malignancy of triple-negative breast cancer by regulating selfrenewal and tumorigenesis of stem cells[J]. Cell Physiol Biochem, 2018, 48(2): 593-604.

[42] Bollig-Fischer A, Marchetti L, Mitrea C, et al. Modeling time-dependent transcription effects of HER2 oncogene and discovery of a role for E2F2 in breast cancer cellmatrix adhesion[J]. Bioinformatics, 2014, 30(21): 303643.

[43] Nguyen-Vu T, Vedin LL, Liu K, et al. Liver x receptor ligands disrupt breast cancer cell proliferation through an E2F-mediated mechanism[J]. Breast Cancer Res, 2013, 15(3): R51.

[44] Shen Q, Uray IP, Li Y, et al. The AP-1 transcription factor regulates breast cancer cell growth via cyclins and E2F factors[J]. Oncogene, 2008, 27(3): 366-77.

[45] Andrechek ER. HER2/Neu tumorigenesis and metastasis is regulated by E2F activator transcription factors[J]. Oncogene, 2015, 34(2): 217-25.

[46] Wu L, de Bruin A, Wang H, et al. Selective roles of E2Fs for ErbB2- and Myc-mediated mammary tumorigenesis[J]. Oncogene, 2015, 34(1): 119-28.

[47] Rawla P, Barsouk A. Epidemiology of gastric cancer: global trends, risk factors and prevention[J]. Prz Gastroenterol, 2019, 14(1): 26-38.

[48] Tarazona N, Gambardella V, Huerta M, et al. Personalised treatment in gastric cancer: Myth or reality[J]? Curr Oncol Rep, 2016, 18(7): 41.

[49] Yang L, Zheng R, Wang N, et al. Incidence and mortality of stomach cancer in China, 2014[J]. Chin J Cancer Res, 2018, 30(3): 291-8.

[50] Hu B, El Hajj N, Sittler S, et al. Gastric cancer: Classification, histology and application of molecular pathology[J]. J Gastrointest Oncol, 2012, 3(3): 251-61.

[51] Orditura M, Galizia G, Sforza V, et al. Treatment of gastric cancer[J]. World J Gastroenterol, 2014, 20(7): 1635-49.

[52] Wang H, Zhang X, Liu Y, et al. Downregulated miR31 level associates with poor prognosis of gastric cancer and its restoration suppresses tumor cell malignant phenotypes by inhibiting E2F2[J]. Oncotarget, 2016, 7(24): 36577-89.

[53] Wen L, Cheng F, Zhou Y, et al. MiR-26a enhances the sensitivity of gastric cancer cells to cisplatin by targeting NRAS and E2F2[J]. Saudi J Gastroenterol, 2015, 21(5): 313-9.

[54] Chen L, Huang X, Chen X. MiR-365 suppresses chol- 
angiocarcinoma cell proliferation and induces apoptosis by targeting E2F2[J]. Oncol Res, 2018, doi: 10.3727/096 $504018 X 15188352857437$.

[55] Dong Y, Zou J, Su S, et al. MicroRNA-218 and microRNA-520a inhibit cell proliferation by downregulating E2F2 in hepatocellular carcinoma[J]. Mol Med Rep, 2015, 12(1): 1016-22.

[56] Fang ZQ, Li MC, Zhang YQ, et al. MiR-490-5p inhibits the metastasis of hepatocellular carcinoma by downregulating E2F2 and ECT2[J]. J Cell Biochem, 2018, 119(10): 8317-24.

[57] Yao Z, Chen Q, Ni Z, et al. Long non-coding RNA differentiation antagonizing nonprotein coding RNA (DANCR) promotes proliferation and invasion of pan- creatic cancer by sponging miR-214-5p to regulate E2F2 expression[J]. Med Sci Monit, 2019, 25: 4544-52.

[58] Mason D, Zhang X, Marques TM, et al. Human papillomavirus 16 E6 modulates the expression of miR-496 in oropharyngeal cancer[J]. Virology, 2018, 521: 149-57.

[59] Tao T, Shen Q, Luo J, et al. MicroRNA-125a regulates cell proliferation via directly targeting E2F2 in osteosarcoma[J]. Cell Physiol Biochem, 2017, 43(2): 768-74.

[60] Gao Y, Ma X, Yao Y, et al. miR-155 regulates the proliferation and invasion of clear cell renal cell carcinoma cells by targeting E2F2[J]. Oncotarget, 2016, 7(15): 20324-37.

Received 21 February 2020, Revised 7 April 2020, Accepted 12 April 2020 